Supporting Information

For

\title{
Synthesis and Characterization of Novel Ferrocene-containing Pyridylamine Ligands and Their Ruthenium(II) Complexes: Electronic Communication through Hydrogen-bonded Amide Linkage
}

\author{
Takahiko Kojima, ${ }^{*}{ }^{1}$ Daisuke Noguchi, ${ }^{2}$ Tomoko Nakayama, ${ }^{2}$ Yuji Inagaki,,${ }^{2, \dagger}$ \\ Yoshihito Shiota, ${ }^{3}$ Kazunari Yoshizawa, ${ }^{3}$ Kei Ohkubo, ${ }^{1}$ and Shunichi Fukuzumi ${ }^{1}$
}

Department of Material and Life Science, Graduate School of Engineering, Osaka University and SORST (JST), Yamada-oka, Suita, Osaka 565-0871, and Department of Chemistry, Faculty of Sciences and Institute for Materials Chemistry and Engineering, Kyushu University, Hakozaki, Higashi-Ku, Fukuoka 812-8581, Japan

E-mail: kojima@chem.eng.osaka-u.ac.jp (T. K.)

${ }^{1}$ Department of Material and Life Science, Osaka University and SORST (JST).

${ }^{2}$ Department of Chemistry, Faculty of Science, Kyushu University.

${ }^{3}$ Institute for Materials Chemistry and Engineering, Kyushu University.

${ }^{\dagger}$ Present address: Department of Applied Quantum Physics, Graduate School of Engineering, Kyushu University, 744 Motooka, Fukuoka 819-0395, Japan 


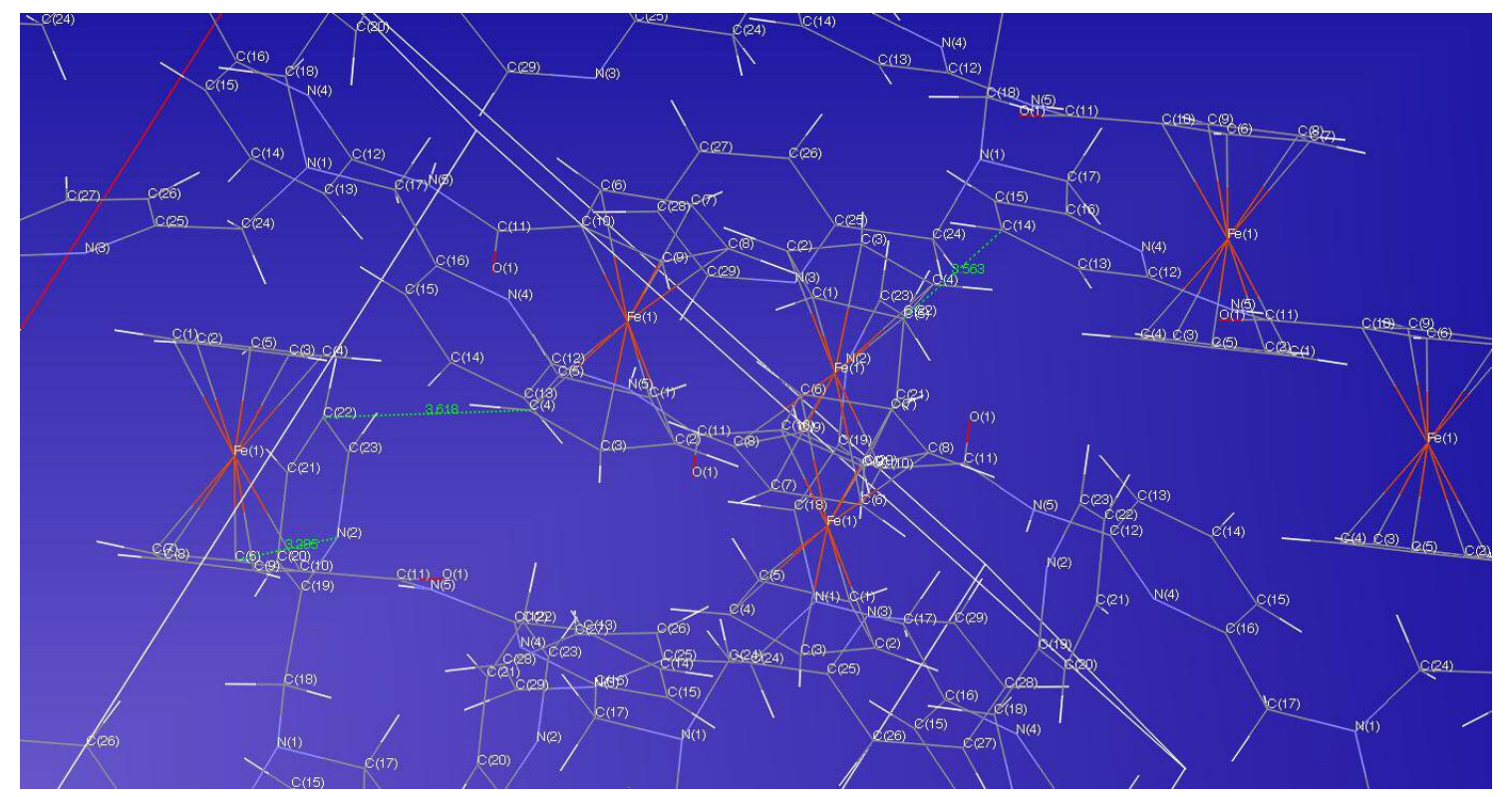

Figure S1. Crystal packing of $\mathbf{L} \mathbf{1}$ to demonstrate intermolecular $\pi-\pi$ interactions. Green dotted lines indicate the interactions with interatomic distances. 


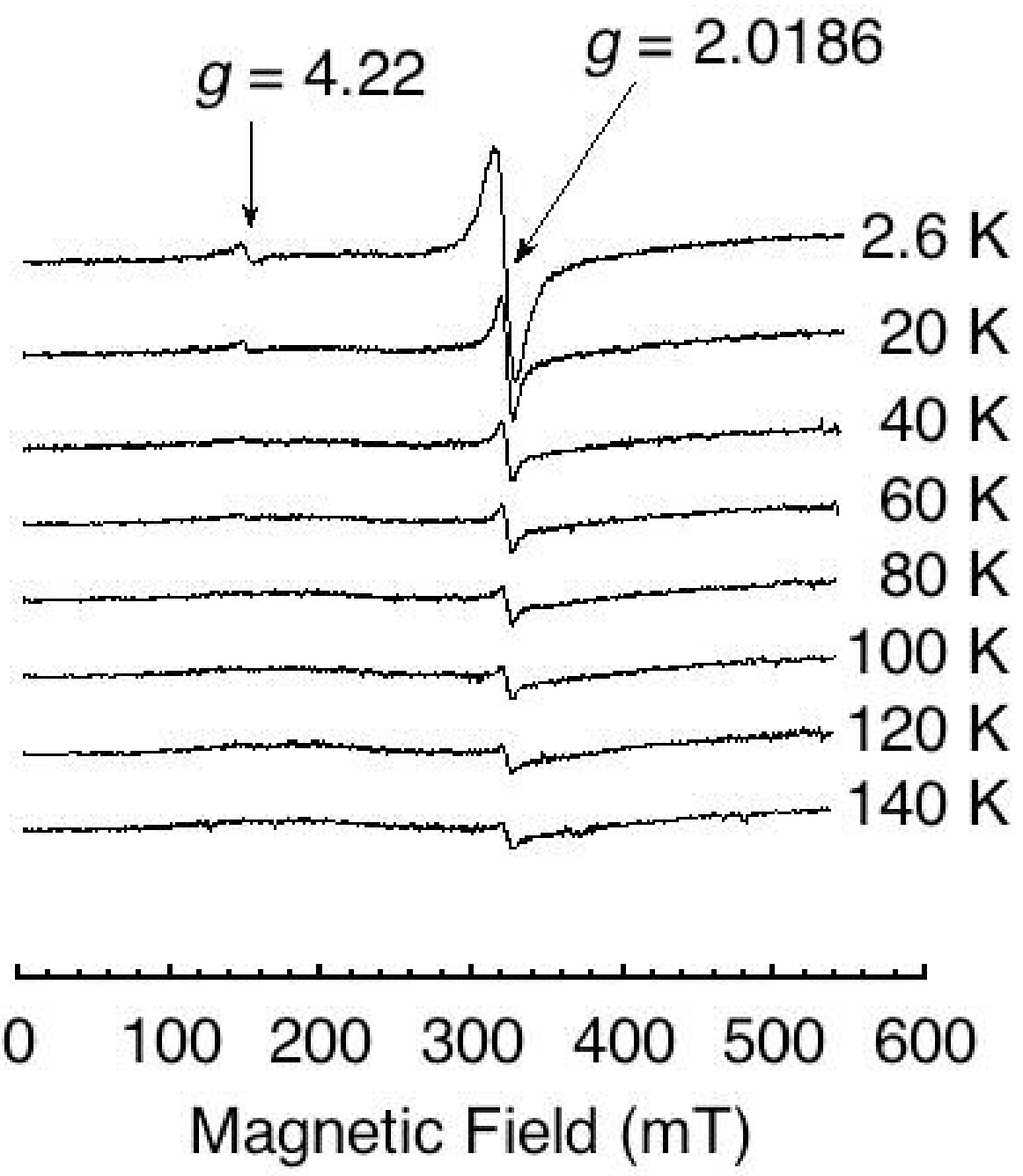

Figure S2. Variable-temperature ESR spectra of $\mathrm{Fc}^{+}$-TPA in a frozen $\mathrm{CH}_{3} \mathrm{CN}$ solution. 
ESR intensity was estimated by the following procedure. In the whole temperature range we measured, both ESR absorptions corresponding to $\Delta m_{S}=1$ and $\Delta m_{S}=2$ transitions were overlapped each other. A typical example of integrated ESR spectrum is shown in Figure S3. To evaluate the net intensity for the signal due to $\Delta m_{S}=2$ transition, therefore, we subtracted the contribution of $\Delta m_{S}=1$ by assuming appropriate polynomial function as demonstrated by the dotted line in Figure S3. Because of the small signal intensity above $120 \mathrm{~K}$, we restricted our analysis to the spectra observed below $103 \mathrm{~K}$. Then, the resultant temperature dependence of the peak intensity is shown in Figure 7, where the longitudinal axis is expressed by the multiplication of the intensity $I$ and the temperature $T$. 


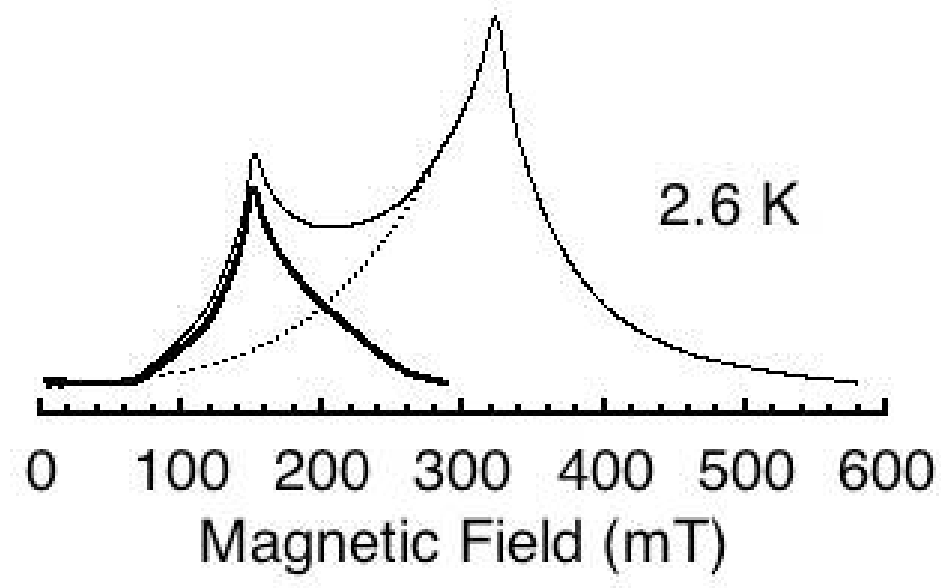

Figure S3. Estimation of peak intensity for the signal due to the $\Delta m_{\mathrm{s}}=2$ transition. 